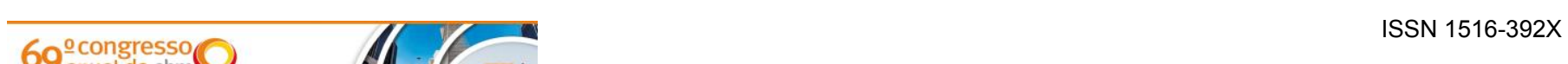

ema: Materiais cerâmicos, compósitos e poliméricos

\title{
SÍNTESE E CARACTERIZAÇÃO DE FERRITA DE NÍQUEL E NÍQUEL-ZINCO PELO MÉTODO DE POLIMERIZAÇÃO DE COMPLEXOS*
}

\author{
Raimison Bezerra de Assis $^{1}$ \\ Samara Melo Valcacer ${ }^{2}$ \\ Camila Cruz da Silva ${ }^{3}$ \\ Ariadne de Souza Silva 4 \\ Maurício Roberto Bomio Delmonte 5 \\ Carlos Alberto Paskocimas 6 \\ Rubens Maribondo do Nascimento ${ }^{7}$ \\ Uílame Umbelino Gomes ${ }^{8}$ \\ Fabiana Villela da Motta $^{9}$
}

\section{Resumo}

Ferritas de níquel-zinco são materiais cerâmicos ferrimagnéticos com estrutura cristalina cúbica do tipo espinélio, pertencente ao grupo espacial $\mathrm{Fd} 3 \mathrm{~m}$, sendo uma solução sólida de composição $\mathrm{NiO}$, $\mathrm{ZnO}$ e $\mathrm{Fe}_{2} \mathrm{O}_{3}$. $\mathrm{O}$ método de polimerização de complexos tem enorme potencial para obtenção de cerâmicas magnéticas, por se tratar de um processo simples, que se origina a partir de materiais com elevado grau de pureza, o que leva à obtenção de pós com elevada homogeneidade química e elevada área superficial. Este trabalho tem como objetivo avaliar as características estruturais e morfológicas das ferritas $\mathrm{Ni}_{0,25} \mathrm{Zn}_{0,75} \mathrm{Fe}_{2} \mathrm{O}_{4}$ e NiFe${ }_{2} \mathrm{O}_{4}$ obtidas através do método polimerização de complexos. A solução foi submetida a uma temperatura final de $120^{\circ} \mathrm{C}$ até a formação da resina, pirolisada a uma temperatura de $350^{\circ} \mathrm{C}$ durante 2 horas e calcinada a $500^{\circ} \mathrm{C}$ durante 2 horas. Os pós resultantes foram caracterizados por difração de raios $X$ (DRX), análises térmicas (TG/ DSC) e microscopia eletrônica de varredura (MEV). Os resultados do DRX das duas composições apresentaram formação de fase majoritária ferrita. Os resultados das micrografias obtidas por MEV mostraram a formação de partículas aglomeradas com placas longitudinais para ambas composições, sendo características microestruturais importantes para aplicação magnética. O método de polimerização de complexos se apresentou como um método promissor para obtenção de pós cerâmicos de ferrita de níquel e níquel-zinco para aplicação magnética.

Palavras-chave: Ferrita Ni; Ferrita Ni-Zn; Pechini; Nanopós magnéticos.

\section{SYNTHESIS AND CHARACTERIZATION OF NICKEL AND NICKEL FERRITE-ZINC BY POLYMERIZATION METHOD OF COMPLEX}

\section{Abstract}

Nickel-zinc ferrites are ferrimagnetic ceramic materials with cubic spinel type crystal structure, space group $\mathrm{Fd} 3 \mathrm{~m}$ belonging to being a solid solution composition of $\mathrm{NiO}, \mathrm{ZnO}$ and $\mathrm{Fe}_{2} \mathrm{O}_{3}$. The complex polymerization method has enormous potential for obtaining magnetic ceramics, because it is a simple process, that originates from materials with high purity, which leads to obtaining powders with high chemical homogeneity and high surface area. This work aims to evaluate the structural and morphological characteristics of ferrites $\mathrm{Ni}_{0,25} \mathrm{Zn}_{0,75} \mathrm{Fe}_{2} \mathrm{O}_{4}$ and $\mathrm{NiFe}_{2} \mathrm{O}_{4}$ obtained by polymerization complex method. The solution was subjected to a final temperature of $120^{\circ} \mathrm{C}$ until the formation of the resin pyrolysed at a temperature of $350^{\circ} \mathrm{C}$ for 2 hours and calcined at $500^{\circ} \mathrm{C}$ for 2 hours. The resulting powders were characterized by X-ray diffraction (XRD), thermal analysis (TG/ DSC) and scanning electron microscopy (SEM). The results of XRD showed the formation of two compositions majority of ferrite phase. The results of SEM micrographs showed the formation of agglomerated particles with longitudinal plates to both compositions being important microstructural characteristics for magnetic application. The method of polymerization of complexes appeared as a promising method to obtain ceramic powders of nickel ferrite and nickel-zinc alloy for magnetic application.

Keywords: Ni ferrite; Ni-Zn ferrite; Pechini; Magnetic Nanopowders.

1 Tecnologia em Fabricação Mecânica, Mestre, Doutorando em Ciência e Engenharia de Materiais, Programa de Pós-graduação em Ciência e Engenharia de Materiais (PPGCEM), Universidade Federal do Rio Grande do Norte (UFRN), Natal, RN, Brasil.

2 Tecnologia em Materiais, Mestre, Doutoranda em Ciência e Engenharia de Materiais, PPGCEM, UFRN, Natal, RN, Brasil.

3 Tecnologia em Fabricação Mecânica, Mestre, Doutoranda em Engenharia Mecânica, PPGEM, UFRN, Natal, RN, Brasil.

4 Engenheira Civil, Doutora, Pós-doutoranda, Laboratório de Materiais Cerâmicos e Metais Especiais (LMCME), UFRN, Natal, RN, Brasil.

5 Químico, Doutor, Professor, Departamento de Engenharia de Materiais, UFRN, Natal, RN, Brasil.

6 Engenheiro de Materiais, Doutor, Professor, Depto. de Engenharia de Materiais, UFRN, Natal, RN, Brasil.

7 Engenheiro Mecânico, Doutor, Professor, Depto. de Engenharia de Materiais, UFRN, Natal, RN, Brasil.

8 Física, Doutor, Professor, Departamento de Física Teórica e Experimental (DFTE), UFRN, Natal, RN, Brasil.

9 Engenheira Química, Doutora, Professora, Depto. de Engenharia de Materiais, UFRN, Natal, RN, Brasil.

\footnotetext{
* Contribuição técnica ao 69ํ Congresso Anual da ABM - Internacional e ao 14ํㅡㄹ ENEMET - Encontro Nacional de Estudantes de Engenharia Metalúrgica, de Materiais e de Minas,21 a 25 de julho de 2014, São Paulo, SP, Brasil.
} 


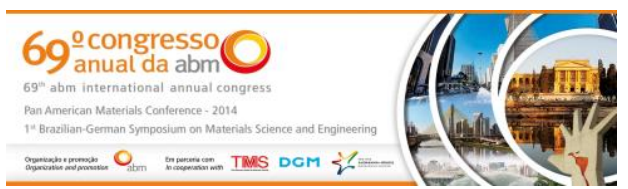

\section{INTRODUÇÃO}

As ferritas $\mathrm{Ni}$ e $\mathrm{Ni}-\mathrm{Zn}$ vêm sendo investigadas de forma ampla e detalhada nos últimos anos, muito tem sido publicado no que se refere as suas propriedades e métodos de obtenção, frente a caracterização em função de diversos fatores, sendo melhoradas com aplicação das novas tecnologias [1]. Ferritas da série Ni-Zn são materiais cerâmicos que apresentam estrutura cristalina do tipo espinélio, pertencente ao grupo espacial $\mathrm{Fd} 3 \mathrm{~m}$, sendo materiais ferrimagnéticos, sendo uma solução sólida, tendo em sua composição química óxidos de $\mathrm{NiO}, \mathrm{ZnO}$ e $\mathrm{Fe}_{2} \mathrm{O}_{3}$. O óxido de ferro na forma de hematita $\left(\alpha-\mathrm{Fe}_{2} \mathrm{O}_{3}\right)$ constitui em torno de $70 \%$ em peso de sua composição. As ferritas $\mathrm{Ni}-\mathrm{Zn}$ com estrutura cúbica são formadas por oito unidades do tipo $\mathrm{AB}_{2} \mathrm{O}_{4}$. $\mathrm{A}$, corresponde a um cátion divalente nos interstícios tetraédricos e $\mathrm{B}$ a um cátion trivalente nos interstícios octaédricos da estrutura cúbica de face centrada formada pelos átomos de oxigênio [2, 3].

As propriedades físico-químicas são tecnologicamente importantes para a indústria, dessa forma, possibilita uma gama de aplicação tecnológica, tais como: catalisadores heterogêneos em processos químicos para reação de deslocamento de vapor de água e oxidação seletiva de CO [4, 5], absorvedores de radiação eletromagnética (MARE) para diferentes faixas de frequência [6, 7], pigmentos [8], ferrofluidos [9] e marcadores magnéticos [10].

Métodos químicos vêm sendo desenvolvidos e aprimorados para obtenção de ferritas, dentre eles, se destaca o Método de Polimerização de Complexos, conhecido também como Método Pechini [11]. O método se baseia na obtenção de poliésteres a partir de citratos metálicos, que após a síntese da solução de citrato, é adicionado um poliálcool, como o etileno glicol, para promover a polimerização. 0 Método de Polimerização de Complexos foi desenvolvido para obter pós de óxidos multicomponentes tendo composição homogênea e alto grau de pureza, como: filmes finos, fibras, materiais dielétricos entre outros, com estequiometria controlada. Este trabalho tem como objetivo avaliar as características estruturais e morfológicas das ferritas $\mathrm{Ni}_{0,25} \mathrm{Zn}_{0,75} \mathrm{Fe}_{2} \mathrm{O}_{4}$ e $\mathrm{NiFe}_{2} \mathrm{O}_{4}$ sintetizadas pelo Método Polimerização de Complexos.

\section{MATERIAIS E MÉTODOS}

Os reagentes de partida foram: ácido cítrico, nitrato de ferro, nitrato de níquel e etilenoglicol para a ferrita $\mathrm{NiFe}_{2} \mathrm{O}_{4}$ e a inclusão do nitrato de zinco para a ferrita $\mathrm{Ni}_{0,25} \mathrm{Zn}_{0,75} \mathrm{Fe}_{2} \mathrm{O}_{4}$. Os reagentes utilizados com o grau de pureza, procedência e os pesos moleculares para os cálculos estequiométricos estão descritos na Tabela 1.

Tabela 1. Reagentes utilizados na síntese pelo Método de Polimerização de Complexos

\begin{tabular}{ccccc}
\hline Reagente & $\begin{array}{c}\text { Fórmula } \\
\text { química }\end{array}$ & Pureza & Procedência & $\begin{array}{c}\text { Peso Molecular } \\
(\mathrm{g} / \mathrm{mol})\end{array}$ \\
\hline Ácido Cítrico $(\mathrm{AC})$ & $\mathrm{C}_{6} \mathrm{H}_{8} \mathrm{O}_{7} \cdot \mathrm{H}_{2} \mathrm{O}$ & $99,5 \%$ & Vetec & 210,14 \\
\hline Nitrato de Ferro & $\mathrm{Fe}\left(\mathrm{NO}_{3}\right)_{3} \cdot 9 \mathrm{H}_{2} \mathrm{O}$ & $98 \%$ & Aldrich & 404,00 \\
\hline Nitrato de Níquel & $\mathrm{Ni}\left(\mathrm{NO}_{3}\right)_{2} \cdot 6 \mathrm{H}_{2} \mathrm{O}$ & $98 \%$ & Vetec & 290,80 \\
\hline Nitrato de Zinco & $\mathrm{Zn}\left(\mathrm{NO}_{3}\right)_{2} \cdot 6 \mathrm{H}_{2} \mathrm{O}$ & $98 \%$ & Aldrich & 297,49 \\
\hline Etileno Glicol (EG) & $\mathrm{C}_{2} \mathrm{H}_{6} \mathrm{O}_{2}$ & $98 \%$ & Synth & 62,07 \\
\hline
\end{tabular}

O ácido cítrico foi dissolvido na água a uma temperatura de $65^{\circ}$ (40\% - AC/ 60\% $E G)$, em seguida adicionados os nitratos de ferro, de níquel e de zinco a uma temperatura de $75^{\circ} \mathrm{C}$, numa proporção 3:1 (AC: Metal), havendo, então a formação

* Contribuição técnica ao $69^{\circ}$ Congresso Anual da ABM - Internacional e ao 14ํㅡㄹ ENEMET - Encontro Nacional de Estudantes de Engenharia Metalúrgica, de Materiais e de Minas,21 a 25 de julho de 2014, São Paulo, SP, Brasil. 


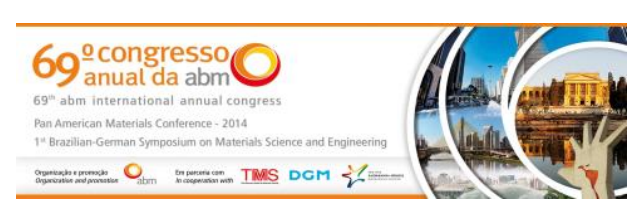

dos citratos metálicos. O etileno glicol foi adicionado por último para promover a polimerização. A síntese foi assistida por agitação e aquecimento constante, conforme Figura 1. A resina polimérica obtida pelo método pechini foi pré-calcinada a $350^{\circ} \mathrm{C}$ durante 2 horas para eliminar parte dos compostos orgânicos, obtendo assim o pó precursor. O pó foi calcinado a $500^{\circ} \mathrm{C}$ durante 2 horas com taxa de aquecimento de $10^{\circ} \mathrm{C} / \mathrm{min}$, resultando no pó cerâmico $\mathrm{NiFe}_{2} \mathrm{O}_{4}$ e $\mathrm{Ni}_{0,25} \mathrm{Zn}_{0,75} \mathrm{Fe}_{2} \mathrm{O}_{4}$.

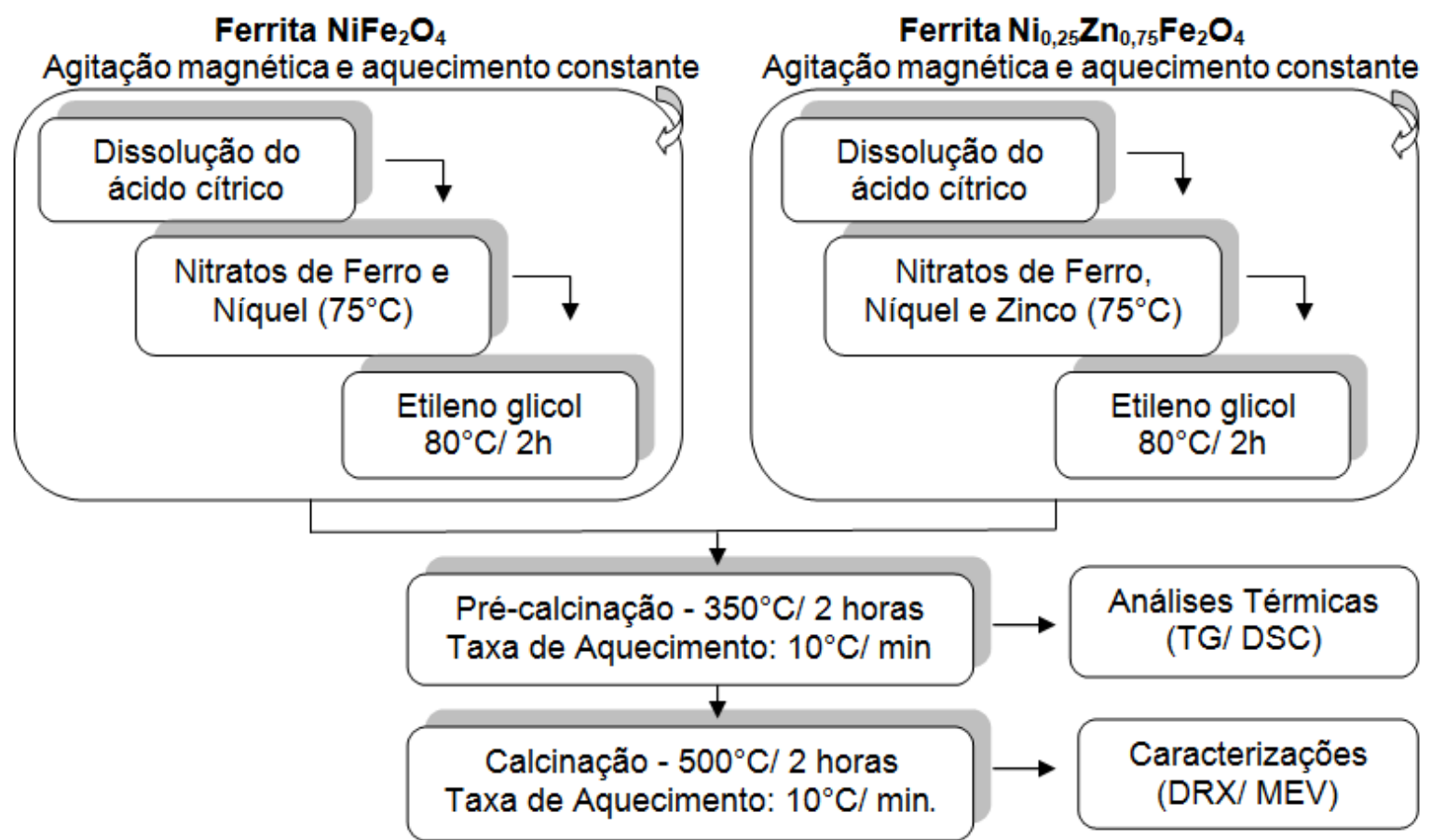

Figura 1. Obtenção das Ferritas $\mathrm{NiFe}_{2} \mathrm{O}_{4}$ e $\mathrm{Ni}_{0,25} \mathrm{Zn}_{0,75} \mathrm{Fe}_{2} \mathrm{O}_{4}$ pelo Método de Polimerização de Complexos.

O pó precursor foi caracterizado pela análise termogravimétrica (TG) e calorimetria exploratória diferencial (DSC) utilizando um analisador termogravimétrico modelo Netzsch STA 449F com massa em torno de 15mg, sob fluxo de ar sintético, taxa de aquecimento de $10^{\circ} \mathrm{C} / \mathrm{min}$ até a temperatura de $950^{\circ} \mathrm{C}$. Os pós das ferritas $\mathrm{NiFe}_{2} \mathrm{O}_{4}$ e $\mathrm{Ni}_{0,25} \mathrm{Zn}_{0,75} \mathrm{Fe}_{2} \mathrm{O}_{4}$ foram caracterizados estruturalmente e morfologicamente por difração de raios $X$ (DRX) e microscopia eletrônica de varredura (MEV), respectivamente. Para análise de $D R X$ foi utilizado um difratômetro de raios $X$ modelo Shimadzu XRD 7000, com radiação $\mathrm{Cu}$ Ka que opera em tubo de alvo de cobre a uma voltagem de $40 \mathrm{kV}$ e $30 \mathrm{~mA}$ de corrente e uma faixa de varredura de 10 a $80^{\circ}$, através da técnica do pó. A micrografias foram obtidas através do microscópio eletrônico de varredura (MEV) modelo TM 3000 Hitachi High Technologies, utilizando tensão de $15 \mathrm{kV}$.

\section{RESULTADOS E DISCUSSÃO}

Os resultados do difratograma de raios $X$ das ferritas calcinadas a $500^{\circ} \mathrm{C} / 2$ horas estão apresentados na Figura 2, evidenciando as fases majoritárias das ferritas com os respectivos planos cristalográficos correspondentes e fases adicionais. Todas as reflexões de Bragg foram correspondentes a estrutura de espinélio da ferrita, os picos se apresentam bem definidos e de alta intensidade.

\footnotetext{
* Contribuição técnica ao 69ำ Congresso Anual da ABM - Internacional e ao 14ํㅡㄹ ENEMET - Encontro Nacional de Estudantes de Engenharia Metalúrgica, de Materiais e de Minas,21 a 25 de julho de 2014, São Paulo, SP, Brasil.
} 

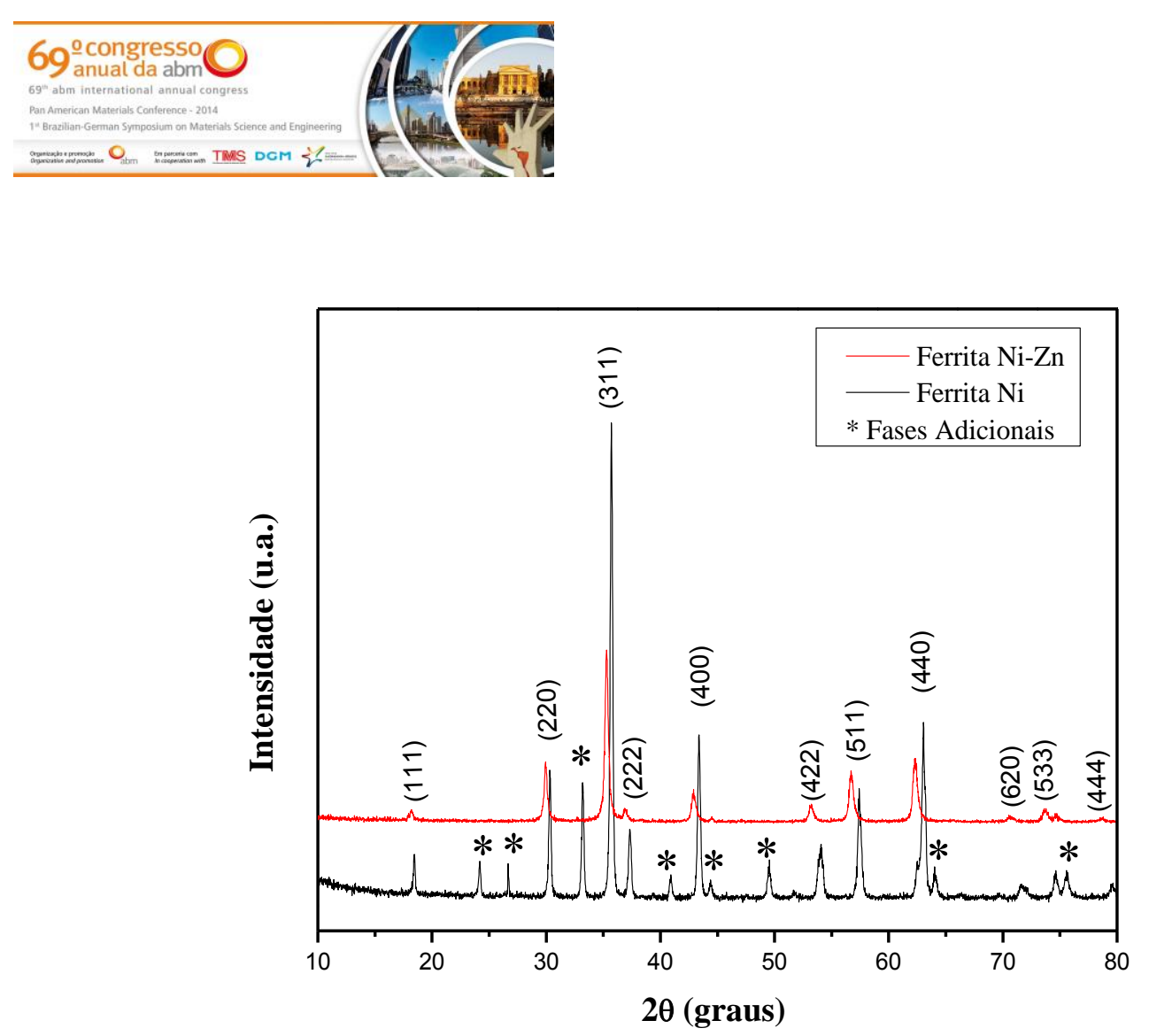

Figura 2. Difratograma de raios $x(\mathrm{DRX})$ das Ferritas $\mathrm{NiFe}_{2} \mathrm{O}_{4}$ e $\mathrm{Ni}_{0,25} \mathrm{Zn}_{0,75} \mathrm{Fe}_{2} \mathrm{O}_{4}$.

A formação de fases adicionais pode ser atribuída ao baixo controle da atmosfera do forno na etapa de calcinação, visto que o ferro é mais estável na forma de óxido, devido ao potencial iônico apresentado. No sistema de ambas as ferritas, o ferro é o formador da rede cúbica de face centrada $[6,12]$. Os íons ferro $\left(\mathrm{Fe}^{2+}\right)$ têm preferência em ocupar os sítios tetraédricos $(A)$ e octaédricos $(B)$ da estrutura de espinélio, já os íons de níquel $\left(\mathrm{Ni}^{2+}\right)$ e zinco $\left(\mathrm{Zn}^{2+}\right)$ têm preferência em ocupar os sítios octaédricos e tetraédricos, respectivamente. Para a ferrita $\mathrm{Ni}$, teoricamente os íons $\mathrm{Fe}^{2+}$ estariam localizados nos dois sítios, e o níquel $\mathrm{Ni}^{2+}$ no sítio octaédrico (B). Já para a ferrita $\mathrm{Ni}-\mathrm{Zn}$, os íons $\mathrm{Fe}^{2+}$ e $\mathrm{Ni}^{2+}$ estariam situados nas posições semelhantes a ferrita de $\mathrm{Ni}$, com a inclusão apenas dos íons $\mathrm{Zn}^{2+}$ nos sítios tetraédricos. Em ambos os sistemas, os íons $\mathrm{Fe}^{2+}$ em excesso nos dois sítios tende a sair do sistema e combinar com o oxigênio da atmosfera do forno na etapa de calcinação para formar hematita $\left(\alpha-\mathrm{Fe}_{2} \mathrm{O}_{3}\right)$ - fase adicional [13]. A temperatura de calcinação $\left(500^{\circ} \mathrm{C}\right)$ conferiu a cristalinidade dos pós obtidos e favoreceu a formação da fase adicional hematita $\left(\alpha-\mathrm{Fe}_{2} \mathrm{O}_{3}\right)$, concordando com os resultados da literatura [12].

Os padrões cristalográficos como tamanho de cristalito (ס), parâmetro de rede (a) e volume da célula unitária $\left(\mathrm{a}^{3}\right)$ são apresentados na Tabela 2.

Tabela 2. Padrões cristalográficos das ferritas $\mathrm{NiFe}_{2} \mathrm{O}_{4}$ e $\mathrm{Ni}_{0,25} \mathrm{Zn}_{0,75} \mathrm{Fe}_{2} \mathrm{O}_{4}$

\begin{tabular}{lccc}
\hline \multicolumn{1}{c}{ Ferrita } & $\begin{array}{c}\text { Tamanho } \\
\text { Cristalito }(\delta)\end{array}$ & $\begin{array}{c}\text { Parâmetro } \\
\text { de rede }\left(\mathrm{a}_{0}\right)\end{array}$ & $\begin{array}{c}\text { Volume } \\
\text { Unitária }\left(\mathrm{a}_{0}{ }^{3}\right)\end{array}$ \\
\hline Ferrita $\mathrm{Ni}\left(\mathrm{NiFe}_{2} \mathrm{O}_{4}\right)$ & $42,45 \mathrm{~nm}$ & $8,33 \AA$ & $578,75 \AA^{3}$ \\
\hline Ferrita $\mathrm{Ni}-\mathrm{Zn}\left(\mathrm{Ni}_{0,25} \mathrm{Zn}_{0,75} \mathrm{Fe}_{2} \mathrm{O}_{4}\right)$ & $35,07 \mathrm{~nm}$ & $8,42 \AA$ & $598,08 \AA^{3}$ \\
\hline
\end{tabular}

O tamanho de cristalito $(\delta)$ foi calculado a partir da linha de alargamento de raios $x$ (pico $d_{311}$ ) usando-se a equação de Scherrer [14], descrita pela equação 1, no qual apresentou tamanho de $42,45 \mathrm{~nm}$ para a ferrita $\mathrm{Ni}$ e 35,07 para a ferrita $\mathrm{Ni}-\mathrm{Zn}$.

\footnotetext{
* Contribuição técnica ao $69^{\circ}$ Congresso Anual da ABM - Internacional e ao 14ํㅡㄹ ENEMET - Encontro Nacional de Estudantes de Engenharia Metalúrgica, de Materiais e de Minas,21 a 25 de julho de 2014, São Paulo, SP, Brasil.
} 


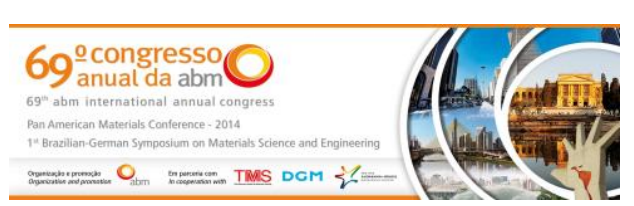

$$
\delta=0,9 \lambda / \beta \cos \theta
$$

A temperatura de calcinação a $500^{\circ} \mathrm{C}$ conduziu o tamanho de cristalino entre 35,07 e 42,45 nanometros. O parâmetro de rede (a) apresentado na Tabela 2 variou de 8,33 a $8,42 \AA$, calculado a partir do software Unit Cell [15], a variação apresentada foi devido a inclusão de íons Níquel na estrutura cúbica do material, bem como proporcionou uma variação do volume da célula unitária de $5,7875 \times 10^{-28}$ e $5,9808 \times 10^{-28} \mathrm{~m}^{3}$. O parâmetro de rede e o volume da célula unitária estão de acordo com trabalhos reportados na literatura $[12,13]$.

A Figura 3 apresenta as curvas das análises térmicas pela (a) análise termogravimétrica (TG) e (b) calometria exploratória diferencial (DSC) do pó precursor, após a pré-calcinação a uma temperatura $350^{\circ} \mathrm{C}$ durante 2 horas.
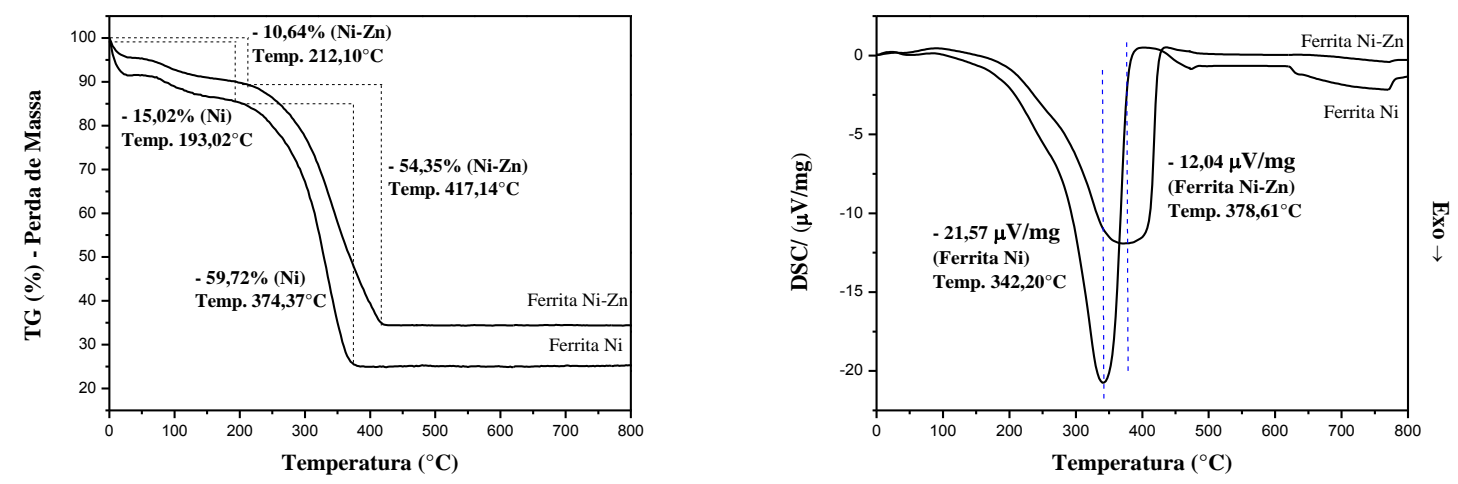

Figura 3. Curva de análises térmicas por TG/ DSC das ferritas Ni e Ni-Zn.

Através da curva, observa-se que o material apresenta a primeira perda de massa de $10,64 \%$ entre 20 e $212,10^{\circ} \mathrm{C}$ aproximadamente para a ferrita $\mathrm{Ni}-\mathrm{Zn}$ e uma perda inicial de $15,02 \%$ entre 20 e $193,02^{\circ} \mathrm{C}$ para a ferrita $\mathrm{Ni}$, no qual para ambas as perdas de massas podem está relacionadas com a evaporação da água e os gases adsorvidos da ferrita [16].

As mudanças mais significativas da perda de massa foram apresentadas em torno de $213,4^{\circ} \mathrm{C}$ até $417,14^{\circ} \mathrm{C}$ (perda de $54,35 \%$ ) para a ferrita $\mathrm{Ni}-\mathrm{Zn}$ e perda de $59,72 \%$ para a ferrita $\mathrm{Ni}$, entre 194,34 e $374,37^{\circ} \mathrm{C}$, no qual estão atribuídas à combustão e a perda de material orgânico. Observa-se que o material é termicamente estável, não havendo picos até a temperatura de $800^{\circ} \mathrm{C}$, o que confirma a alta estabilidade térmica da ferrita $\mathrm{Ni}$ e $\mathrm{Ni}-\mathrm{Zn}$, evidenciando o início da cristalização do material. Através da curva DSC, observa-se picos exotérmicos significativos em torno de $378,61^{\circ} \mathrm{C}$ e $342,20^{\circ} \mathrm{C}$ para a ferrita $\mathrm{Ni}-\mathrm{Zn}$ e $\mathrm{Ni}$, respectivamente, atribuído ao início da eliminação dos compostos orgânicos conforme o TG, apresentando uma maior perda de energia para a ferrita $\mathrm{Ni}(21,57 \mu \mathrm{V} / \mathrm{mg})$, justificado pela alta liberação de energia no processo de eliminação de orgânico e à combustão.

A Figura 4 apresenta as micrografias dos pós obtidos. A temperatura de calcinação influenciou no tamanho de cristalito e na larga distribuição de partículas. Observa-se a formação de blocos porosos (não densos) oriundos do processo da calcinação (eliminação de material orgânico), com características irregulares, constituídas de aglomerados de partículas finas com porosidade interparticular.

\footnotetext{
* Contribuição técnica ao $69^{\circ}$ Congresso Anual da ABM - Internacional e ao 14ํㅡㄹ ENEMET - Encontro Nacional de Estudantes de Engenharia Metalúrgica, de Materiais e de Minas,21 a 25 de julho de 2014, São Paulo, SP, Brasil.
} 

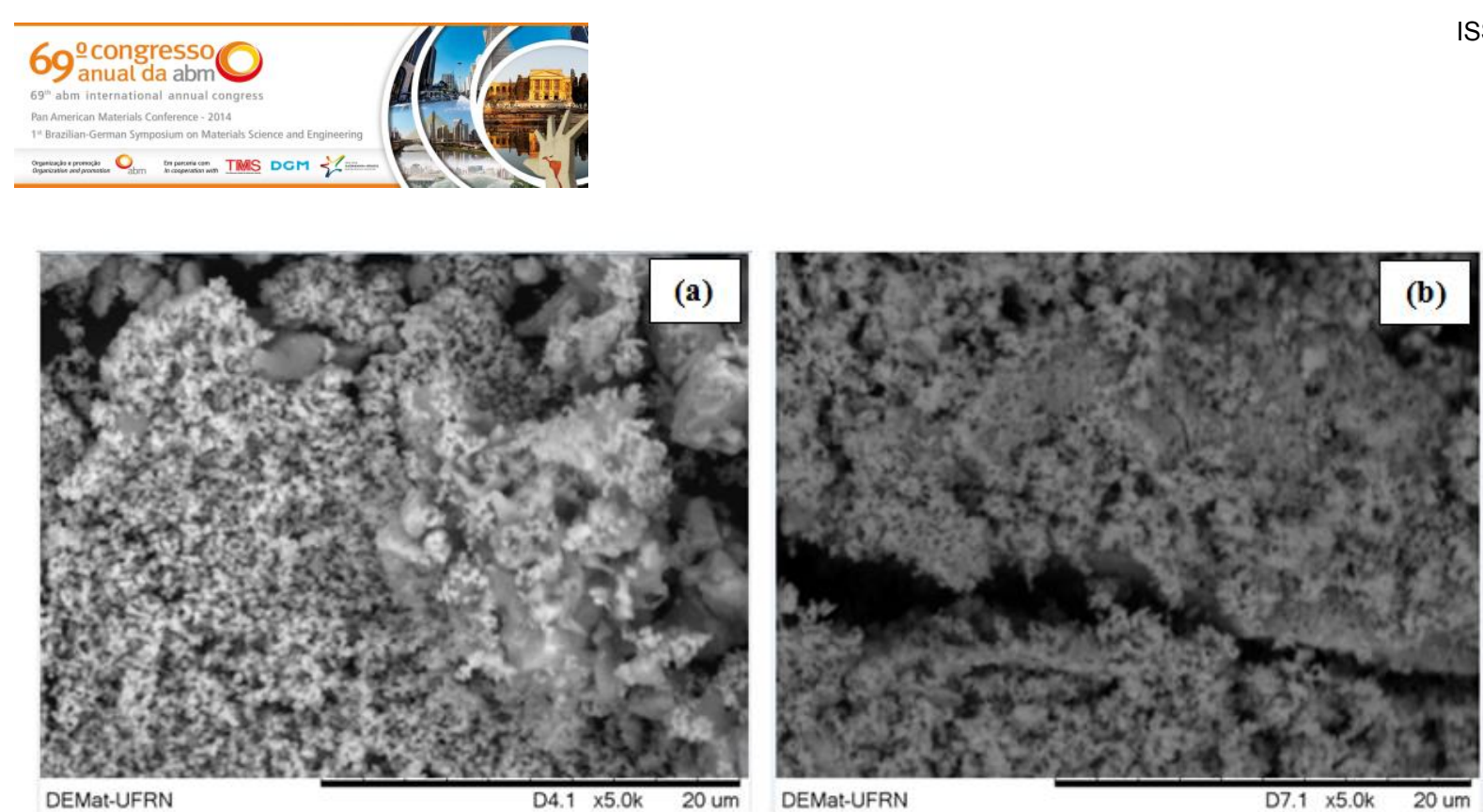

Figura 4. Micrografia das ferritas (a) $\mathrm{NiFe}_{2} \mathrm{O}_{4}$ e (b) $\mathrm{Ni}_{0,25} \mathrm{Zn}_{0,75} \mathrm{Fe}_{2} \mathrm{O}_{4}$ obtidas por MEV.

Esses blocos porosos são definidos morfologicamente como aglomerados de características moles ou friáveis (constituídas de ligações fracas) e de fácil desaglomeração. Observa-se placas com superfícies rugosas e longitudinais, como resultado do efeito do aquecimento por gradiente térmico na morfologia do material.

\section{CONCLUSÃo}

A obtenção de pós de ferritas $\mathrm{NiFe}_{2} \mathrm{O}_{4}$ e $\mathrm{Ni}_{0,25} \mathrm{Zn}_{0,75} \mathrm{Fe}_{2} \mathrm{O}_{4}$ pelo método de polimerização de complexos se mostrou eficiente quanto a qualidade das propriedades estruturais e morfológicas do material. Resultados do DRX confirmaram a formação da fase ferrita (espinélio) em temperatura e tempo relativamente baixos, conduzidos pelos resultados das análises térmicas, indicando a temperatura inicial de cristalização e a preservação das características morfológicas deste material. Conclui-se que o método de polimerização de complexos apresenta vantagens significativas para obtenção de ferritas, como o bom controle estequiométrico, alta homogeneidade química, qualidade da microestrutura e morfologia, evidenciando uma técnica promissora.

\section{Agradecimentos}

Os autores agradecem o apoio financeiro da CAPES, CNPq e ao Programa de Pósgraduação em Ciência e Engenharia de Materiais (PPGCEM) da Universidade Federal do Rio Grande do Norte pela disponibilidade de recursos de materiais.

\section{REFERÊNCIAS}

1 Brito VLO. Ferritas Ni-Zn: breve revisão sobre o processo convencional de fabricação e as propriedades permeabilidade magnética e constante dielétrica. Cerâmica. 2006; 52 : 221-231.

2 Rane KS, Verenkar VSM.; Swant PY. Hidrazine method of synthesis of $\mathrm{Y}-\mathrm{Fe}_{2} \mathrm{O}_{3}$ useful in ferrites preparation. Part IV - preparation abd charaterization of magnesium ferrite, $\mathrm{MgFe}_{2} \mathrm{O}_{4}$ from $\mathrm{y}-\mathrm{Fe}_{2} \mathrm{O}_{3}$ obtained from hidrazinated iron oxyhydroxides and iron (II) carboxylato-hidrazinates. Journal of Materials Science Materials Eletronics. 1994; 10: 133-140.

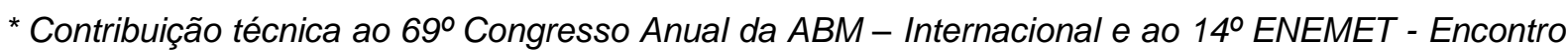
Nacional de Estudantes de Engenharia Metalúrgica, de Materiais e de Minas,21 a 25 de julho de 2014, São Paulo, SP, Brasil.
} 


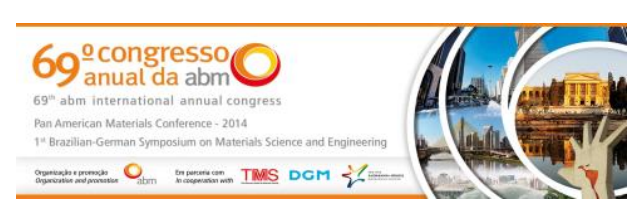

3 Moura AEG. Síntese, sinterização e caracterização de ferritas à base de Ni-Zn. Dissertação de Mestrado, Natal - RN: Universidade Federal do Rio Grande do Norte, 2008.

4 Santos PTA et al. Synthesis of a $\mathrm{NiFe}_{2} \mathrm{O}_{4}$ Catalyst for the Preferential Oxidation of Carbon Monoxide (PROX). Journal of Alloys and Compounds, 2009; 483: 399-401.

5 Santos PTA et al. Evaluation of $\mathrm{NiFe}_{2} \mathrm{O}_{4}$ Spinel, Synthesized by Combustion Reaction, as a Catalyst for Selective CO Oxidation. Materials Science Forum, 2010; 660-661: 771-776.

6 Bezerra DC. et al. Compósitos de PA 6/ferrita $\mathrm{NiFe}_{2} \mathrm{O}_{4}$. Parte 1 - Caracterização estrutural e morfológica. Revista Eletrônica de Materiais e Processos, 2009; 4: 01-11.

7 Bezerra DC. et al. Development of Polyamide6/Ferrite Composites for Absorbers of Electromagnetic Radiation. Materials Science Forum, 2010; 660-661: 922-927.

8 Costa ACFM. et al. Brown pigment of the nanopowder spinel ferrite prepared by combustion reaction. Journal of the European Ceramic Society. 2008; 28: 2033-2037.

9 Schettino Junior MA. Obtenção e caracterização de nanopartículas magnéticas inseridas em materiais carbonosos porosos a partir da decomposição do pentacarbonil ferro. Dissertação de Mestrado, Vitória - ES: Universidade Federal do Espírito Santo, Centro de Ciências Exatas, Programa de Pós-Gradução em Física, 2009.

10 Roca AG. Preparación de nanopartículas magnéticas uniformes y de alta cristalinidad para biomedicina. Instituto de Ciência de Materiales de Madrid, CSIC - Departamento de Materiales Particulados Universidad Complutense de Madrid Departamento de Química Física I Madrid, febrero 2009.

11 PECHINI, M. P., U.S. Patent $n^{\circ}$ 3.330.697, 1967.

12 Olhero SM. et al. Co-precipitation of a Ni-Zn ferrite precursor powder: Effects of heat treatment conditions and deagglomeration on the structure and magnetic properties. Journal of the European Ceramic Society, 2012; 32: 2469-2476.

13 Kothawale MM. et al. Characterization and Magnetic Properties of Nanoparticle $\mathrm{Ni}_{1-\mathrm{x}} \mathrm{Zn}_{\mathrm{x}} \mathrm{Fe}_{2} \mathrm{O}_{4}$ Ferrites Prepared Using Microwave Assisted Combustion Method. Journal Supercond Nov Magn, 2012; 25: 1907-1911.

14 Azároff LV. Elements of X-ray crystallography. McGraw-Hill Book Company, 1968.

15 UNITCELL-97 program [T.J.B Holland, S.A.T. Redfem, Unit cell refinement from powder diffraction data: the use of regression diagnostics, Miner. Mag. 61(1997) 65-67].

16 Rahimi M. et al. The effect of zinc doping on the structural and magnetic properties of $\mathrm{Ni}_{1-x} \mathrm{Zn}_{x} \mathrm{Fe}_{2} \mathrm{O}_{4}$. J Mater Sci. 2013; 48: 2969-2976..

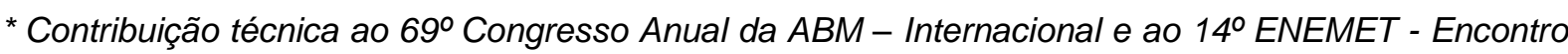
Nacional de Estudantes de Engenharia Metalúrgica, de Materiais e de Minas,21 a 25 de julho de 2014, São Paulo, SP, Brasil.
} 